Einführung in das Schwerpunktthema

\title{
Global Governance und Umweltpolitik
}

Von Ulrich Petschow ie umfassende Liberalisierung der Märkte und damit zusammenhängend die Globalisierungstendenzen haben zu Veränderungen der Balance der Handlungsmöglichkeiten von staatlichen und wirtschaftlichen Akteuren geführt. Jüngstes Beispiel der Liberalisierungsbemühungen sind die Verhandlungen über ein Multilaterales Investitionsabkommen der OECDStaaten, das als „Charta der Rechte und Freiheiten der transnationalen Konzerne“ bezeichnet wird (das Institut für Ökologische Wirtschaftsforschung erstellt zu diesem Thema aktuell ein Gutachten). Die Diskussion wird nicht nur in Deutschland vom Schlagwort der umfassenden (positiven) Handlungsunfähigkeit der nationalen Akteure beherrscht. Als Folge der wirtschaftlichen Globalisierung wird das existierende Gefüge zwischen den demokratisch gewählten Regierungen und privatwirtschaftlichen Akteuren mehr und mehr durch ökonomische Macht (ungewählt und unrepräsentativ) beeinflußt. Die Globalisierung der Umweltwirkungen des wirtschaftlichen Handelns ist hingegen schon deutlich geworden bevor das Schlagwort der wirtschaftlichen „Globalisierung" kreiert wurde. Beispielsweise ist das Ozonloch nicht allein durch den Nationalstaat bekämpfbar.

Damit ist faktisch eine doppelte Globalisierung bedeutsam (1):

- die umfassende Liberalisierung und damit Globalisierung der Märkte,

- die Globalisierung der Umweltprobleme.

Zwar sind nicht alle Umweltprobleme grundsätzlich globaler Natur und deren Lösung damit von internationaler Kooperation abhängig (vgl. hierzu den Beitrag von Kirchgässner), aber in der aktuellen Diskussion wird deutlich, daß auch manche nationalen Umweltprobleme unter dem zunehmenden Wettbewerbsdruck nicht mehr effektiv gelöst werden (können).

In beiden Fällen der Globalisierung wird deutlich, daß der Nationalstaat als bisher zentraler Akteur der Wirtschafts- und Umweltpolitik an Durchsetzungsfähigkeit verloren hat, weil sich wichtige sozio-politische Akteure seinem Einfluß zunehmend entziehen können.
Die Liberalisierung der Märkte durch Beseitigung von Handelshemmnissen und Wettbewerbsbeschränkungen wird häufig als negative oder auch marktschaffende Integration beschrieben. Positive Integration wird hingegen als gestaltende Politik begriffen. Sie ist zwar darauf ausgerichtet, einen einheitlichen Markt zu schaffen, entwickelt dabei aber zugleich den „Rahmen“ für das Wirtschaften auf einer höheren, nicht mehr nationalstaatlichen Ebene. Insofern kann sie auch als marktkorrigierend charakterisiert werden. Generell ist aber ein Bias zugunsten der Liberalisierung gegenüber dem Aufbau von Regulierungen bzw. Rahmensetzungen festzustellen. Dies gilt von der Welthandelsorganisation WTO, deren Position deutlich stärker als die multilateraler Umweltschutzabkommen ist, bis - abgeschwächt - zur Europäischen Union (EU).

Aus diesen Zusammenhängen ergibt sich, daß sich der souveräne Staat nunmehr inmitten eines Beziehungsgeflechtes von internationalen Regimen und Organisationen wiederfindet, die sich etabliert haben bzw. noch etabliert werden müssen. Traditionelle nationale Lösungswege werden damit in Frage gestellt. Gleichzeitig erhöhen sich die Regulierungsnotwendigkeiten. Selbst in Bezug auf die ungehemmte wirtschaftliche Globalisierung werden mittlerweile kritische Stimmen laut (2). Die Entwicklung eines Ordnungsrahmens, die insbesondere im Umweltbereich erforderlich ist, muß jedoch die abnehmende Handlungsfähigkeit der Staaten berücksichtigen.

Dennoch ist die Frage geeigneter politischer Strategien weiterhin umstritten. Scharpf unterscheidet drei Ansätze (3):

- In der neoliberalen Literatur zur politischen Ökonomie der Globalisierung wird der Abbau der Außengrenzen befürwortet. Der damit ausgelöste Wettbewerb der politischen Systeme beschränke die Staatsmacht und erhöhe die (angestrebte) Freiheit der Märkte gegenüber politischen Interventionen.

- Ein weiterer Argumentationsstrang sieht in dem Verlust nationalstaatlicher Steuerungspotentiale gegenüber der globalisierten Ökonomie ein Ende der Demokratie herannahen. Er zielt daher auf die Wiederherstellung nationalstaatlicher Macht.

- Ein dritter Ansatz sieht die Möglichkeit, den Verlust der Handlungsfähigkeit nationaler Politik durch den Aufbau supranationaler Regelungskompetenzen auszugleichen.

Folgı man dem letztgenannten Argument, werden vor allem Fragen des geeigneten Übergangs hin zu transnationalen Beziehungen aufgeworfen. Die ordoliberale Position eines starken „rahmensetzenden“ Staates hilft an dieser Stelle nicht weiter. Es geht vielmehr darum, ein Vakuum zu fuillen, das zur Regulierung oder auch nur zur Regelung von ungewünschten Wirkungen von freien Austauschprozessen beiträgt. Folglich besteht die Notwendigkeit der Selbstorganisation jenseits der Nationalstaaten, um eine positive Integration zu initiieren und zu verstärken. Ohne die grundsätzliche Möglichkeit einer positiven Integration wäre ,governance without government" (4) zum Scheitern verurteilt (5).

An diesem Punkt setzt das Konzept der Global Governance an (siehe Kasten). Dieses greift die Probleme der abnehmenden nationalen Handlungsmöglichkeit und der begrenzten internationalen Problemlösungsfähigkeit auf und entwickelt Ansatzpunkte für Governance-Strukturen, die eine Vielzahl von Akteuren einbeziehen. Die identifizierten Probleme gelten gerade auch für die Umweltpolitik, in der die zivilgesellschaftlichen Akteure schon immer von besonderer Bedeutung waren. Inwiefern kann diese sinnvoll mit dem Konzept der Global Governance verkniipft werden? Ziel dieses Schwerpunktes von Ökologisches Wirtschaften ist dabei weniger die Vertiefung dieses Konzeptes an sich. Vielmehr sollen einige Ansatzpunkte einer möglichen (Weiter-)Entwicklung von transnationalen Strukturen im Umweltbereich aufgezeigt werden.

\section{- Die Beiträge im Überblick}

Im ersten Beitrag gibt Gebhard Kirchgässner einen Überblick über Bedingungen internationaler Umweltpolitik und nationale Handlungsmöglichkeiten im Rahmen der Globalisierung. Im Anschluß daran werden zwei europäische Erfahrungen dargestellt. Auf der einen Seite analysiert Christoph Knill die Marktliberalisierung und gleichzeitige Entwicklung eines eigenständigen Handlungsfeldes „Umweltpolitik“ in der EU. Er macht deutlich, unter welchen Rahmenbedingungen die Entwicklung einer Umweltordnung 
möglich ist. Der Beitrag von Rainer Durth geht von den Erfahrungen der Rheinsanierung aus und zeigt plastisch die Möglichkeiten einer Governance jenseits staatlichen Handelns auf. So hat gerade die Einbeziehung weiterer (nicht-staatlicher) Akteure - durch die Eröffnung von Handlungsmöglichkeiten - zur Verbesserung der Umweltsituation am Rhein geführt. Die Abbildungen in diesem Beitrag veranschaulichen die Entwicklung eines Beziehungsgeflechtes nach Einschränkung des staatlichen Handlungsmonopols. Sie verdeutlichen damit beispielhaft die Situation unter veränderten Governance-Strukturen.

Am Beispiel des FCKW-Problems wird von Reiner Grundmann die Bedeutung unterschiedlicher Akteure und insbesondere konkurrierender Akteursnetzwerke für die Lösung von Umweltproblemen aufgezeigt. Dabei spielt gerade in Bereichen, in denen unter Unsicherheit entschieden werden muß, die Wissenschaft eine wichtige Rolle.

Die Klimakonferenz in Kioto, die zweifelsohne weit hinter den ökologischen Erfordernissen des Klimaschutzes zurückgeblieben ist, wird in ihren Implikationen von Wilbelm Althammer beschrieben. Es wird deutlich, daß es sich bei handelbaren Emissionsrechten, deren Einführung perspektivisch angestrebt wird, um ein hoch- komplexes Instrument handelt. Zunächst müssen überhaupt erst die Bedingungen für einen Markt geschaffen werden, wobei aktuelle und zukünftige Verteilungswirkungen von besonderer Bedeutung sind. Einmal etabliert kann dieser Markt aber auch seine eigene Dynamik entfalten.

Die beiden abschließenden Beiträge beschreiben die Problematik aus Sicht wichtiger nichtstaatlicher Akteure. Elmar Altvater, Achim Brunnengräber und Heike Walk untersuchen durchaus kritisch die Bedeutung, die NichtRegierungs-Organisationen (NGOs) als zivilgesellschaftlichen Akteuren im Rahmen von Global Governance zukommen kann. Peter Fuchs beschäftigt sich mit transnationalen Konzernen und deren Bedeutung für die Umweltpolitik; vor allem analysiert er die Beeinflussung der Agen$\mathrm{da}$ internationaler Verhandlungen durch diese Unternehmen.

In der Summe wird deutlich, daß es „die“ Lösung der globalen Umweltprobleme nicht gibt und auch nicht die Akteure, die allein in der Lage wären, eine Lösung zu realisieren. Vielmehr müssen die Umweltprobleme differenziert betrachtet und je nach Situation der Handlungsrahmen identifiziert und diesem angemessene Lösungsansätze ergriffen werden. Entscheidend dürfte jedoch sein, solche Ansätze zu verfolgen, die allgemein die Kooperationsfähigkeit erhöhen.

\section{Anmerkungen}

(1) Vgl. für eine umfassende Analyse Petschow, U., K.Hübner, S.Dröge, J.Meyerhoff: Nachhaltigkeit und Globalisierung. Berlin u.a. 1998 sowie

OECD: Economic Globalisation and the Environment, Paris 1997.

(2) Vgl. z.B. Rodrik, Dani: Has Globalisation gone too far? Institute for International Economics, Washington 1997.

(3) Scharpf, F.W.: Balancing Positive and Negative Integration: The Regulatory Options for Europe. MPlfG Working Paper 8/1997.

(4) Rosenau, J.N., E.O.Czempiel (Hg.): Governance without Government. Order and Change in World Politics. New York 1992.

(5) Vgl. Zürn, M.: Die Implementation internationaler Umweltregime und "positive Integration". MPIfG Discussion Paper 3/1996.

Der Autor
Ulrich Petschow ist wissenschattlicher Mitarbeiter
am lÖW.
Kontakt: 10̈W, Giesebrechtstr. 13, 10629 Berlin.
Tel. 030/8845940, Fax 030/8825439,
E-mail: mailbox@ioew.b.eunet.de

D. Von Achim Brunnengräber und Heike Walk er Begriff der Global Governance wurde wesentlich von der 1991 ouf Initiative von Willy Brandt gebil: deten "Commission on Global Governonce" geprägt. Das 28 -köpfige, internationale Expertengremium hatte es sich zur Aufgabe gemacht, die Möglichkeiten der Ausgestaltung eines effektiveren Systems der Weltsicherheit, einer nachhaltigen Entwicklung und der universellen Ausbreitung der Demokratie nach dem Ende des Kalten Krieges auszuleuchten und Vorschläge zur Regierbarkeit der Welt (also nicht einzelner Nationalstaaten oder von Zusam. menschlüssen von Nationalstaaten) zU unterbreiten. 1994 wurde der Endbericht der Kommission in englischer (1), ein Jahr später von Stiftung für Entwicklung und Frieden in deutscher Sprache vorgelegt (2).

Nach der Definition der Kommission ist "Governance [...] die Gesamtheit der zahlreichen Wege, ouf denen Individuen sowie offentliche und private Institutionen ihre gemeinsomen Angelegenheiten regeln. Es hondelt sich um einen kontinuierlichen Prozeb, durch den kontroverse oder unterschiedliche Interessen ausgeglichen werden und kooperatives Handeln initiiert werden kann. Der Begriff umfaßt sowohl formelle Institutionen und mit Durchsetzungsmacht versehene Herrschaftssysteme als auch informelle Regelungen, die von Menschen und Institutionen vereinbart oder als im eigenen Interesse angesehen werden" (S.4).

\section{Stichwort: Global Governance}

Der größte Teil des Berichts der Kommission orientiert sich an der "Notwendigkeit von Visionen" (S.14). Er unterbreitet Hondlungsempfehlungen und eröffnet einen breiten Interprefationsspielraum. Auf die Interessengegensätze und Konfliktpotentiale der verschiedenen Akteure, die über die unterschiedlichsten (Macht-) Ressourcen verfügen, wird von der Kommission freilich nicht eingegangen. Eine solche Herangehensweise würde der Logik der Kommission widersprechen, die nach "Werten für die Nachbarschaft in der einen Welt" oder nach einem "glo. balen Bürgerethos" sucht. Auch wird Globol Governance nicht mit Weltregierung gleichgesetzt.

Eine deutsche Übersetzung des Ausdrucks ist schwierig. Sofern nicht das englische Original beibehalten wird, findet sich häufig, wie auch in der deutschen Übersetzung des Berichts, der aufgrund seiner Konnotation mit einem starken Staat eher problematische Begriff "Welt. ordnungspolitik".

Trotz aller Unklarheiten, die hinsichtlich des Projektes noch bestehen, zielt Global Governance auf neve Politikfelder, die inzwischen in der globalen Politikarena bearbeitet werden müssen; vor allem ouf den unauflöslichen Zusammenhang von Umwelt- und Entwicklungspolitik, wie er im "Brundtland-Report" und dann ouf der UNCED in Rio de Janeiro 1992 themotisiert worden ist. Global Governonce zielt aber auch auf eine erweiterte Zahl von (neven) Akteuren, wie sie auch in der Agenda 21 benonnt werden. Dazu zählen neben internationalen Organisationen und den Regierungen auch Marktakteure, soziale Organisationen (wie Arbeitgeber und Gewerkschaften) und Institutionen (wie z.B. das Bildungswesen) - und nicht zuletzt NGOs, die hier gleichermafen als „ziviler Stützpfeiler”, als „unabhängige Kontrollorgane", "Frühwarnsysteme" und "Informationsquellen" angesehen werden (S.281f). Schließlich bezieht sich Global Governance auf die im Zuge der Globalisierung entwickelten neven Formen von Politik.

\section{Anmerkungen}

(1) Commission on Global Governance: Our Global Neigh. bourhood. Cambridge 1994

(2) Stiftung Entwicklung und Frieden ( $\mathrm{Hg}$.): Nachbarn in Einer Welt. Der Bericht der Kommission für Weltordnungspolitik. The Commission on Global Governance, Bonn 1995.

Kontakte zu den Autorlnnen siehe Seite 25 
(c) 20I0 Authors; licensee IÖW and oekom verlag. This is an article distributed under the terms of the Creative Commons Attribution Non-Commercial No Derivates License (http://creativecommons.org/licenses/by-nc-nd/3.o/), which permits unrestricted use, distribution, and reproduction in any medium, provided the original work is properly cited. 